\title{
Single-agent cytarabine is insufficient for the treatment of human mantle cell lymphoma in mouse xenograft model
}

\author{
M. KLANOVA ${ }^{1,2, *}$, T. SOUKUP ${ }^{3}$, J. MOLINSKY ${ }^{1,2}$, L. LATECKOVA ${ }^{1,2}$, P. VOCKOVA ${ }^{1,2}$, M. ALAM ${ }^{1}$, J. ZIVNY ${ }^{1}$, M. TRNENY ${ }^{2}$, P. KLENER ${ }^{1,2}$ \\ ${ }^{1}$ Institute of Pathological Physiology, First Faculty of Medicine, Charles University in Prague, U Nemocnice 5, Prague, Czech Republic; ${ }^{2}$ First \\ Department of Medicine- Clinical Department of Haematology, General University Hospital and First Faculty of Medicine, Charles University \\ in Prague, U Nemocnice 2, Prague, Czech Republic; ${ }^{3}$ Institute of Histology and Embryology, Medical Faculty Hradec Kralove, Charles University, \\ Simkova 870, Hradec Kralove, Czech republic
}

${ }^{*}$ Correspondence: magda.klanova@lf1.cuni.cz

Received October 13, 2015 / Accepted May 9, 2016

\begin{abstract}
Mantle cell lymphoma is an aggressive type of B-cell non-Hodgkin lymphoma with adverse prognosis. It was demonstrated that alternation of CHOP and DHAP chemotherapy improved outcome of mantle cell lymphoma patients. However, which components of DHAP, cisplatin, cytarabine, or both, were responsible for the improved outcome remained unclear. To answer this question, antitumor efficacies of equally toxic doses of cytarabine, cisplatin, and three different combinations were compared in vivo using mouse xenograft models of mantle cell lymphoma. We demonstrated that cisplatin, alone or with cytarabine, is significantly superior to single-agent cytarabine in both eliminating lymphoma cells and suppressing their proliferation rate.
\end{abstract}

Key words: mantle cell lymphoma (MCL), cisplatin, high-dose cytarabin, DHAP

Mantle cell lymphoma (MCL) is an aggressive type of B-cell non-Hodgkin lymphoma characterized by frequent relapses and adverse prognosis. Therapy of MCL has been for decades based on anthracyclines and alkylating agents, namely on the CHOP (cyclophosphamide, doxorubicine, vincristine and prednisone) or CHOP-like regimen. In recent years the outcome of patients with MCL has been improved by doseintensification of anthracyclines, addition of high-dose araC (HDAC) to the multi-agent chemotherapy [1], incorporation of anti-CD20 antibody rituximab (R) into induction and maintenance [2-3], and consolidation with high-dose therapy and autologous stem cell transplantation (ASCT) [4]. Recently, several studies showed that DHAP regimen (dexamethasone, high-dose araC and cisplatin) is highly effective

\footnotetext{
Abbreviations: Mantle cell lymphoma (MCL), cisplatin (Pt), cytarabine (araC), high-dose araC (HDAC), CHOP (cyclophosphamide, doxorubicine, vincristine and prednisone), methotrexate (MTX), immunohistochemical (IHC), bone marrow (BM), twice daily (BID), overall survival (OS), DHAP (dexamethasone, high-dose araC and cisplatin), complete remission (CR), time to treatment failure (TTF), autologous stem cell transplantation (ASCT), rituximab (R), NOD-SCID-gamma (NSG), HyperCVAD (cyclophosphamide, vincristine, doxorubicin and prednison).
}

as the frontline therapy of MCL patients [5-7]. Lefrere et al demonstrated that 4 cycles of DHAP were capable to convert insufficient responses (partial remissions or stable diseases) induced by 4 cycles of CHOP to complete remissions (CR) in as high as $84 \%$ of patients (22 of 26 patients) [5]. Recently, the randomized trial of The European Mantle Cell Network demonstrated that alternation of R-CHOP and R-DHAP increased significantly $\mathrm{CR}$ rates and time to treatment failure (TTF) compared to R-CHOP only [6]. Based on these results implementation of HDAC into induction regimen became the standard of care for all newly diagnosed younger MCL patients. The enthusiasm for HDAC in front-line therapy of MCL led even to attempts to avoid other chemotherapy agent in the front-line setting. However, the latest Nordic Lymphoma Group Mantle Cell Lymphoma 5 trial that evaluated "single"-agent HDAC (in combination with rituximab, but not other genotoxic agent standardly used in front-line therapy of MCL) was prematurely terminated because of insufficient efficacy [8]. Results from these clinical trials raised a crucial question, which of the two agents, Pt or araC (or both), actually contributed to the improved outcome of MCL patients. Despite that most clinicians (in our opinion) 
originally favored cytarabine (araC) over cisplatin $(\mathrm{Pt})$, the recent Nordic Lymphoma Group trial 5 questioned this assumption. The main goal of this study was to identify, which of the two cytostatic components of the DHAP regimen, araC or Pt (or both), represents a key contributor of the improved clinical efficacy observed in newly diagnosed MCL patients treated with alternation of R-CHOP and R-DHAP compared to R-CHOP-only [6].

\section{Materials and methods}

MCL cell lines and culture conditions. Jeko-1 was obtained from the German Collection of Microorganisms and Cell Cultures, Hbl-2 was a kind gift of Prof. Martin Dreyling (University of Munich, Germany). Cell lines were cultured in Iscove's modified Dulbecco's medium supplemented with $15 \%$ fetal bovine serum and $1 \%$ penicillin/streptomycin at $37^{\circ} \mathrm{C}$ in $5 \% \mathrm{CO}_{2}$.

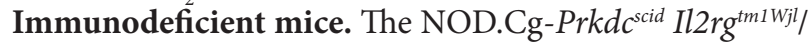
SzJ mice (referred to as NOD-SCID-gamma (NSG)) were purchased from The Jackson Laboratory (Bar Harbor, Maine, USA). All animals were housed and maintained in a pathogen free environment in individually ventilated cages and provided with sterilized food and water. The experiment design was approved by the institutional animal care and use committee.

Experimental therapy of MCL xenografts. Jeko-1 and Hbl-2 cell line-based mouse models of MCL were established in the same way as previously described [9]. Briefly, $8-16$ week old female NSG mice were inoculated with $1 \times 10^{6}$ Jeko- 1 or Hbl-2 cells, and divided into 6 treatment cohorts (8-10 animals/cohort). Treatment was initiated in the middle of the estimated overall survival (OS) of untreated controls (which was day 18 and day 16 for Jeko- 1 and Hbl-2-xenografted mice, respectively). The treatment was derived from the DHAP regimen. $\mathrm{Pt}$ and araC were administered on two consecutive days. Both agents were administered intraperitoneally (i.p.) as follows: 1 . control group treated with PBS only; 2 . single-agent cisplatin $240 \mu \mathrm{g}$; 3. single-agent cytarabine $10 \mathrm{mg}$ twice daily (BID) 12 hours apart; 4 . three different combinations of both drugs designated as $\mathrm{C} 1$ ( $\mathrm{Pt} 160 \mathrm{mcg}+\operatorname{araC} 2 \mathrm{mg} \mathrm{BID}), \mathrm{C} 2(\mathrm{Pt}$ $80 \mathrm{mcg}+\operatorname{araC} 3.5 \mathrm{mg}$ BID), C3 (Pt $40 \mathrm{mcg}+\operatorname{araC} 5 \mathrm{mg}$ BID). All treatment approaches were pre-tested to be equally toxic causing $10-20 \%$ early toxic deaths within 7 days of the drug administration. When untreated controls developed signs of terminal disease, two mice from each cohort were subject to immunohistochemical (IHC) analysis of the selected murine organs. Remaining animals were euthanized by cervical dislocation when developed hint-leg paralysis or generalized inability to thrive. Differences in OS among treatment cohorts were calculated using Kaplan-Meier survival estimates set-up with the Graph-Pad Prism software.

Immunohistochemistry. Murine organs were removed, fixed in $4 \%$ formalin for 3 days, cut into tissue blocks, embedded in paraffin, and sectioned into transversal and longitudinal 6-7 $\mu \mathrm{m}$ thick slices. Sections were stained with hematoxylin and eosin and Masson's blue trichrome. IHC staining for human CD20(L26) and Ki67/MIB1 (DakoCytomation) was performed. Antigen retrieval was done in citrate buffer solution $(0.291 \%$ sodium citrate, $0.05 \%$ Tween $20, \mathrm{pH} 6.0)$ using microwave for $3 \times 5$ minutes. Standard ABC (Avidin Biotin Complex) method was used for the detection with $\mathrm{DAB}$ (3,3'-Diaminobenzidine). Mayer's hematoxylin was used for counterstaining. Images were obtained using Olympus BX51 equipped with a DP25 digital camera. Data was analyzed by Image-Pro Plus 5.1 software within 20 samples from different organ areas (40 visual fields were observed). Experiments were carried out in duplicates (two mice for IHC analysis). The mean percent infiltration of murine tissues (bone marrow (BM) or spleen) with MCL cells (i.e. the percent of the area, which is occupied by CD20-positive or Ki67-positive cells) was calculated by the Image-Pro Plus 5.1 software and is shown (Figure 1B, Table 1).

\section{Results}

Mouse xenograft models of human aggressive MCLs were used to compare head-to-head anti-lymphoma efficacies of equally toxic doses of single-agent araC compared to singleagent $\mathrm{Pt}$, and three different combinations of $\mathrm{araC}$ and $\mathrm{Pt}$ (C1-C3) [9]. Lymphoma-specific survival (defined as the time from lymphoma inoculation to lymphoma-caused demise of experimental animals) was significantly $(\mathrm{p}<0.05)$ prolonged in cohorts treated with single-agent $\mathrm{Pt}$, and $\mathrm{C} 1$ combination compared to the other cohorts (Figure 1A, B). In addition, IHC analysis of the bone marrow (and the spleen in case of Jeko-1 -bearing mice) demonstrated that mice from these treatment

Table 1. Results of immunohistochemical analysis showing percent infiltration (CD20) of murine bone marrow (BM) and spleen with MCL cells, and proliferation rate (Ki67) of MCL cells.

\begin{tabular}{llllcccccc}
\hline & & & & Ctrl & Pt & araC & C1 & C2 \\
\hline & & BM & CD20 & $86.7 \pm 2.8$ & $10.3 \pm 1.8$ & $44.5 \pm 3.2$ & $0.5 \pm 0.4$ & $30.9 \pm 1.6$ & $45.7 \pm 7.8$ \\
Jeko-1 & & & Ki67 & $84.9 \pm 3.2$ & $1.4 \pm 0.2$ & $2.5 \pm 2.1$ & $1.6 \pm 1.6$ & $3.1 \pm 1.9$ & $6.8 \pm 5.1$ \\
& \% Infiltration (CD20) & \multirow{2}{*}{ Spleen } & CD20 & $53.3 \pm 1.9$ & $4.7 \pm 1.4$ & $25.6 \pm 0.6$ & $1.7 \pm 1.2$ & $20.0 \pm 8.3$ & $27.0 \pm 1.6$ \\
& and proliferation (Ki67) & & Ki67 & $21.6 \pm 2.1$ & $1.6 \pm 0.7$ & $13.5 \pm 1.2$ & $1.5 \pm 0.4$ & $6.7 \pm 4.6$ & $16.0 \pm 3.1$ \\
\multirow{2}{*}{ Hbl-2 } & & BM & CD20 & $95.2 \pm 4.8$ & $19.2 \pm 7.7$ & $29.9 \pm 5.4$ & $21.8 \pm 5.2$ & $37.6 \pm 6.1$ & $41.0 \pm 8.5$ \\
& & & Ki67 & $89.1 \pm 5.0$ & $0.5 \pm 0.0$ & $14.2 \pm 6.4$ & $3.7 \pm 1.6$ & $21.5 \pm 4.1$ & $28.5 \pm 5.3$ \\
\hline
\end{tabular}



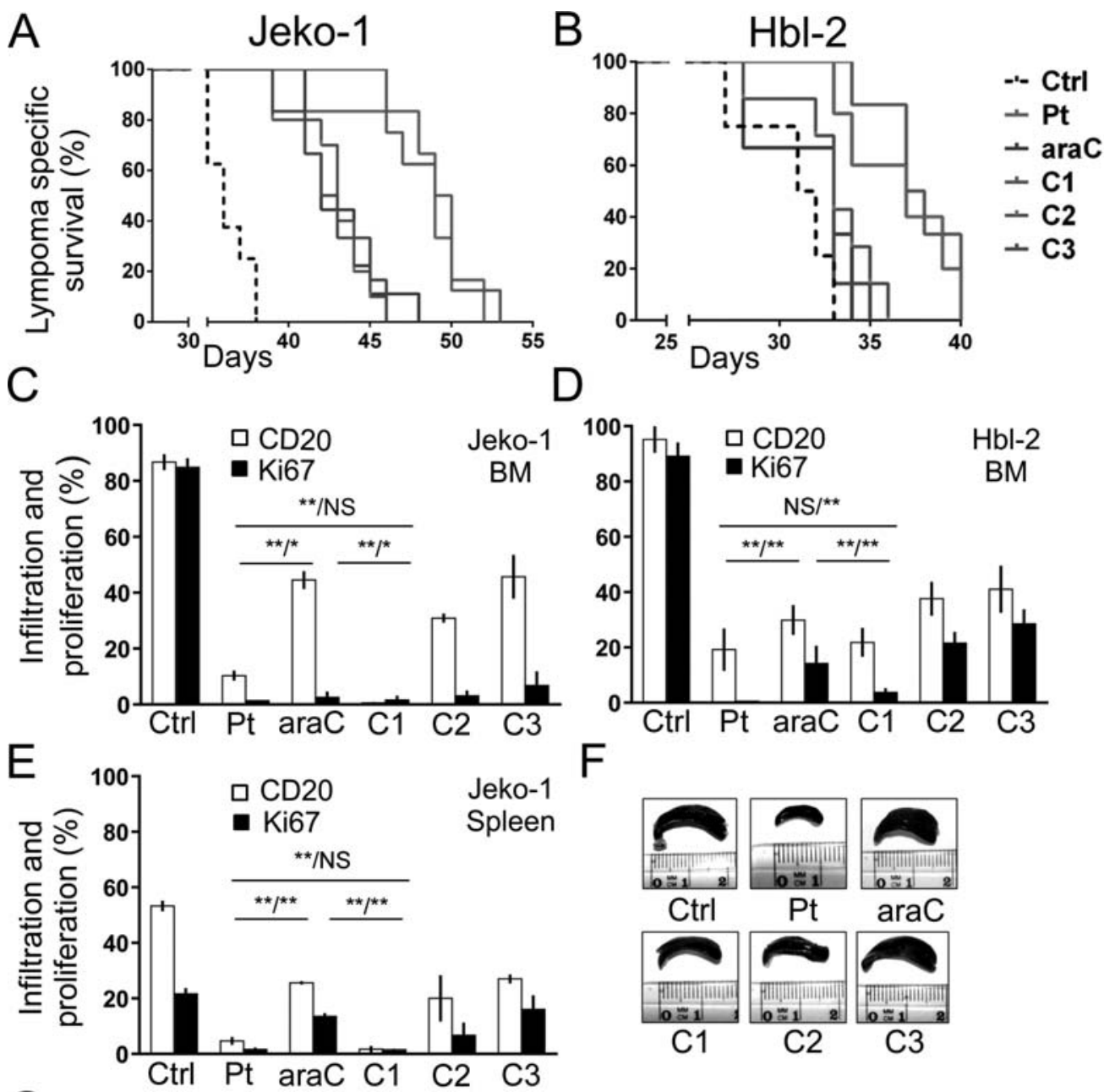

$\mathrm{F}$
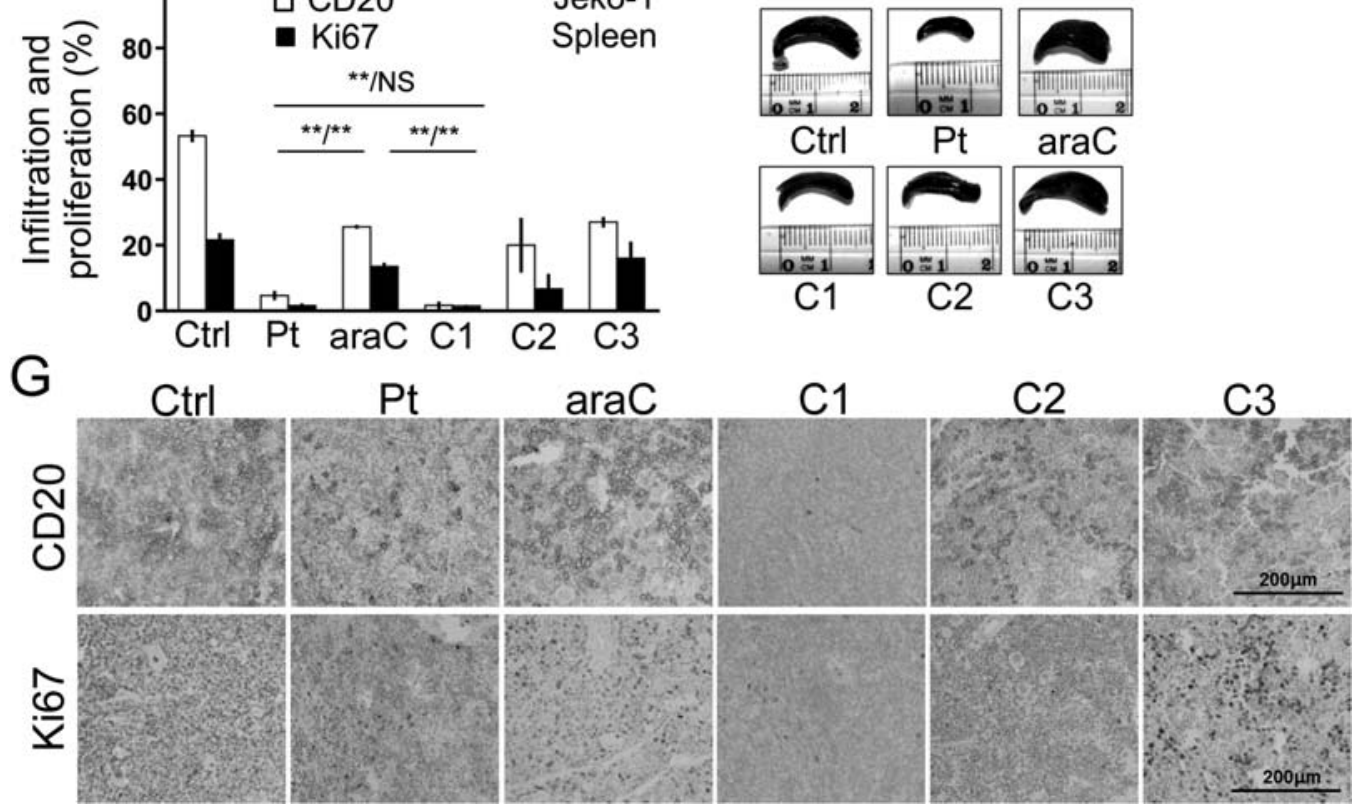

Figure 1. Anti-lymphoma efficacy of araC compared to $\mathrm{Pt}$, and three different combinations of both drugs in mouse xenograft model of MCL

$A-B$ Kaplan-Meier survival curves showing lymphoma specific survival of individual treatment cohorts of Jeko-1 $(A)$ and Hbl-2 (B) xenografted mice. Single-agent cisplatin $(\mathrm{Pt})$ and the $\mathrm{Cl}$ combination significantly $(\mathbf{p}<0.05)$ prolonged lymphoma specific survival in Jeko-1 and Hbl-2 xenografted mice compared both to untreated mice (Ctrl) and to the other cohorts. $C$ - $E$ Results of immunohistochemical analysis showing percent infiltration of murine bone marrow (BM) and spleen with MCL cells (anti-human CD20, white columns), and proliferation rate (Ki67, black columns) of the MCL cells. All 5 treatment approaches significantly $(\mathbf{p}<0.001)$ decreased the extent of infiltration (either by \% of CD20- or Ki67-positive cells) of murine BM or spleen of Jeko-1 and Hbl-2 xenografted mice compared to untreated controls (Ctrl). Combination of Pt and araC (C1) significantly decreased the extent of infiltration (CD20-positive cells) of murine BM or spleen compared to single agent approaches in Jeko-1 xenografted mice. In case of $\mathrm{Hbl-2}$ xenografted mice either the combination of Pt and $\mathrm{AraC}(\mathrm{C1})$ or single-agent Pt significantly decreased the extent of infiltration (CD20-positive cells) of murine BM compared to single-agent AraC. P values are shown for CD20/Ki67, NS= not significant $(p \geq 0.05),{ }^{\star} p<0.05$ and $\geq 0.001,{ }^{* *} p<0.001$.

$F$ Murine spleens removed from the Jeko-1 xenografted mice, both from the untreated animals (Ctrl) and from the mice treated with indicated agent(s). $G$ Immunohistochemical analysis (anti-human CD20 and Ki67) of murine spleen of Jeko-1 xenografted mice (untreated controls (Ctrl) and the mice treated with indicated agent(s). 
cohorts (Pt and C1) had both the lowest organ infiltration with human MCL cells (i.e. number of CD20-positive cells), and the most profound suppression of MCL cell proliferation rate (i.e. number of Ki67/MIB1-positive cells, see Figure 1C-E, G and Table 1). Accordingly, spleens isolated from mice treated with either Pt or $\mathrm{C} 1$ combination were smaller compared to those isolated from the other cohorts (Figure 1F). Our data indicate that single-agent $\mathrm{Pt}$, or the combination of $\mathrm{Pt}$ and araC appear superior to equally toxic doses of single-agent araC in eliminating aggressive MCL cells.

\section{Discussion}

Large body of evidence suggests that araC-based strategies are superior to standard CHOP-like-based-only regimen. In addition to combinations of araC and Pt (DHAP), other strategies were tested in attempt to improve outcome of MCL patients. Romaguera et al demonstrated that alternating RHyperCVAD (cyclophosphamide, vincristine, doxorubicin and prednison) and R-high-dose-methotrexate (MTX) / HDAC induced higher CR and prolonged OS compared to historical controls [2]. Another standardly used treatment strategy is the Nordic Regimen induction immunochemotherapy with rituximab and dose intensified CHOP (maxiCHOP) alternating with R-HDAC. R-maxiCHOP/RHDAC regimen followed by high dose therapy with ASCT induced high CR rates and the 6-year overall and progression-free survival of $70 \%$ and $66 \%$, respectively [3].

As there are currently no randomized trials that would directly compare these different approaches, it remains elusive, which of the agents or strategies are superior in improving outcome of MCL patients: whether hyper-fractionation of cyclophosphamide, dose-intensification of anthracyclines, addition of MTX, addition of Pt, addition of HDAC, or combination of more factors. To answer this question in extenso is far beyond the scope of this manuscript. Here, we focused on the simple question, which of the two agents in the DHAP regimen, $\mathrm{Pt}$ or araC (or both) are key contributors of improved efficacy of this regimen. Recently, the Nordic Lymphoma Group MCL trial 5 was prematurely terminated because of insufficient antilymphoma efficacy of front-line therapy based solely on high-dose araC in combination with rituximab [8]. To simulate the situation in patients with aggressive MCL disease, we used mouse xenograft models of aggressive metastatic human MCL, and subjected the MCLbearing mice to equally toxic therapies consisting of $\mathrm{Pt}$, araC and three different combinations of $\mathrm{Pt}+$ araC. Equal toxicity of individual treatments was absolute prerequisite that enabled us to compare their anti-lymphoma efficacy. We found that either single-agent $\mathrm{Pt}$ or $\mathrm{C} 1$ combination (containing the highest dose of Pt compared to $\mathrm{C} 2$ or $\mathrm{C} 3$ combination) belonged to the most potent treatment approaches. Single-agent araC and C3 combination (the combination with the highest dose of araC) were less effective suggesting araC monotherapy did not effectively suppress aggressive MCL xenografts. In conclusion using these preclinical models we confirmed the results of the Nordic Lymphoma Group trial 5 concluding that single-agent araC is not appropriate treatment for biologically aggressive $\mathrm{MCL}$ in the clinical grounds. In translation the DHAP regimen appears superior to single-agent HDAC-based strategies. The results also indicate that $\mathrm{Pt}$ remains, despite its well-known toxic side-effects, a powerful cytostatic agent, at least in case of aggressive forms of MCL.

Acknowledgements: All in vivo experiments were performed at the Centre for Experimental Biomodels, 1st Faculty of Medicine, Charles University in Prague. IGA-MZ NT/13072-4, AZV 1527757A, UNCE 204021, PRVOUK P24/LF1/3, PRVOUK 27/LF1/1, GACR14-19590S.

\section{References}

[1] KHOURI IF, ROMAGUERA J, KANTARJIAN H, PALMER JL, PUGH WC et al. Hyper-CVAD and high-dose methotrexate/cytarabine followed by stem-cell transplantation: an active regimen for aggressive mantle-cell lymphoma. J Clin Oncol 1998; 16: 3803-3809.

[2] ROMAGUERA JE, FAYAD L, RODRIGUEZ MA, BROGLIO KR, HAGEMEISTER FB et al. High rate of durable remissions after treatment of newly diagnosed aggressive mantle-cell lymphoma with rituximab plus hyper-CVAD alternating with rituximab plus high-dose methotrexate and cytarabine. J Clin Oncol 2005; 23: 7013-7023. http://dx.doi.org/10.1200/ ICO.2005.01.1825

[3] GEISLER CH, KOLSTAD A, LAURELL A, ANDERSEN NS, PEDERSEN LB et al. Long-term progression-free survival of mantle cell lymphoma after intensive front-line immunochemotherapy with in vivo-purged stem cell rescue: a nonrandomized phase 2 multicenter study by the Nordic Lymphoma Group. Blood 2008; 112: 2687-2693. http://dx.doi. org/10.1182/blood-2008-03-147025

[4] DREYLING M, LENZ G, HOSTER E, VAN HOOF A, GISSELBRECHT C et al. Early consolidation by myeloablative radiochemotherapy followed by autologous stem cell transplantation in first remission significantly prolongs progression-free survival in mantle-cell lymphoma: results of a prospective randomized trial of the European MCL Network. Blood 2005; 105: 2677-2684. http://dx.doi.org/10.1182/blood-2004-10-3883

[5] LEFRERE F, DELMER A, SUZAN F, LEVY V, BELANGER C et al. Sequential chemotherapy by CHOP and DHAP regimens followed by high-dose therapy with stem cell transplantation induces a high rate of complete response and improves event-free survival in mantle cell lymphoma: a prospective study. Leukemia 2002; 16: 587-593. http://dx.doi.org/10.1038/sj.leu.2402406

[6] HERMINE O, HOSTER E, WALEWSKI J, RIBRAG V, BROUSSE $\mathrm{N}$ et al. Alternating Courses of $3 \mathrm{x}$ CHOP and $3 \mathrm{x}$ DHAP Plus Rituximab Followed by a High Dose ARA-C Containing Myeloablative Regimen and Autologous Stem Cell Transplantation (ASCT) Increases Overall Survival When Compared to 6 Courses of CHOP Plus Rituximab Followed by Myeloablative Radiochemotherapy and ASCT in Mantle Cell Lymphoma: Final Analysis of the MCL Younger Trial of 
the European Mantle Cell Lymphoma Network (MCL net). ASH Annual Meeting Abstracts 2002; 120: 151.

[7] LE GOUILL S, CALLANAN M, MACINTYRE E, DELFAULARUE M-H, BODET-MILIN C et al. Clinical, Metabolic and Molecular Responses After 4 Courses of R-DHAP and After Autologous Stem Cell Transplantation for Untreated Mantle Cell Lymphoma Patients Included in the LyMa Trial, a Lysa Study. ASH Annual Meeting Abstracts 2012; 120: 152.

[8] LAURELL A, KOLSTAD A, JERKEMAN M, RATY R, GEISLER CH. High dose cytarabine with rituximab is not enough in first-line treatment of mantle cell lymphoma with high proliferation: early closure of the Nordic Lymphoma Group Mantle Cell Lymphoma 5 trial. Leuk Lymphoma 2014; 55: 1206-1208. http://dx.doi.org/10.3109/10428194 .2013 .825906

[9] KLANOVA M, SOUKUP T, JAKSA R, MOLINSKY J, LATECKOVA L et al. Mouse models of mantle cell lymphoma, complex changes in gene expression and phenotype of engrafted MCL cells: implications for preclinical research. Lab Invest 2014; 94: 806-817. http://dx.doi.org/10.1038/ labinvest.2014.61 